\title{
ОРГАНИЗАЦИОННАЯ ГИБКОСТЬ КАК ДИНАМИЧЕСКАЯ СПОСОБНОСТЬ БОЛЕЕ ВЫСОКОГО ПОРЯДКА
}

\author{
(C) 2020 Громова Елизавета Алексеевна \\ ассистент, Высшая инженерно-экономическая школа \\ Санкт-Петербургский политехнический университет Петра Великого, Россия, Санкт-Петербург \\ E-mail: lizaveta-90@yandex.ru
}

Наступление Четвертой промышленной революции ознаменовалось тем, что изменения стали основными характеристиками нового времени. Это означает высокий уровень неопределенности и нестабильности в бизнес-среде. Особое значение приобретает знание механизмов, позволяющих предприятиям выявлять изменения во внешней среде, а также способность адаптироваться к ним. Предлагается рассмотрение организационной гибкости в рамках структуры динамических способностей. Делается вывод, что организационная гибкость - это наивысшее развитие динамических способностей, как результат интеграции ключевых компетенций.

Ключевые слова: организационная гибкость, динамические способности, ключевые компетенции, промышленное предприятие, неопределенность, Четвертая промышленная революция.

В настоящее время промышленные предприятия всё чаще сталкиваются с турбулентной средой, с сильными дозами динамизма, сложности и неопределенности. Эти условия привели к возникновению гиперконкурентных рынков, представляющих серьезную угрозу для выживания компаний. В этом контексте особую актуальность приобретает знание механизмов, позволяющих предприятиям выявлять изменения во внешней среде, а также способность адаптироваться к ним и предлагать надлежащие ответные меры, поскольку эти знания могут привести предприятия к достижению большего успеха за счет использования новых возможностей и новых источников конкурентных преимуществ. Поэтому концепция организационной гибкости представляется ключевым вопросом, касающимся выживания и успеха промышленного предприятия.

Базируясь на идеях научной группы Д.Тиса [10], можно рассматривать организационную гибкость, как способность организации эффективно перераспределять и перенаправлять ресурсы с целью создания и сохранения стоимости деятельности с более высокой доходностью в зависимости от того, что диктуют внутренние и внешние обстоятельства. Предприятия, обладающие этой способностью, стремятся управлять неопределенностью на стороне предложения и корректировать стратегию, если это необходимо. Достижение организационной гибкости - это не универсальное решение. В одном случае, дости- жение имеет смысл, в другом случае, лучший подход - это строить или покупать оборудование общего назначения или диверсифицировать клиентскую базу. Важно, что способности, требуемые для реагирования на негативные события, часто отличаются от тех, которые необходимы, чтобы воспользоваться преимуществом от позитивных явлений. Требования организационной гибкости зависят от контекста. Например, на стабильных рынках бывает более выгодно оптимизировать основные операции и достичь эффективности как раз за счет овладения этой способностью. Однако, когда речь идет о глубокой неопределенности, организационная гибкость становится неотъемлемым атрибутом промышленного предприятия. Автором исследования предлагается рассмотрение данного понятия в рамках структуры динамических способностей (ДС).

Динамические способности определяют, как предприятие интегрирует, создает, реконфигурирует внутренние и внешние компетенции для решения проблем с изменяющейся бизнессредой. Этот класс способностей подкрепляется организационными и управленческими компетенциями, формированием среды и разработкой бизнес-моделей, которые касаются новых возможностей и угроз. Таким образом, динамические способности идентифицируют способность предприятия внедрять инновации, адаптироваться к изменениям и создавать изменения, которые благоприятны для потребителей 
и неблагоприятны для конкурентов. По мнению Д.Тиса [11], к микрооснованиям динамических способностей могут быть отнесены:

- «ощущение» (способности, заключающиеся в определении и осмыслении возможностей и угроз, видении предпринимательских возможностей);

- «захват» (способности заинтересовывать, удерживать и использовать возможности);

- «трансформация» (способности, состоящие в развитии, защите и рекомбинировании материальных и нематериальных активов предприятия, а также смене бизнес-модели).

Эти микрооснования имеют важное значение для того, чтобы предприятие могло поддерживать конкурентоспособность в долгосрочной перспективе по мере смены клиентов, конкурентов и технологий. Взаимодействие с каждым из них является непрерывным или полунепрерывным, но необязательно последовательным. Упрощенная структура динамических способностей представлена на рисунке 1.

Динамические способности могут быть аналитически отделены от формулировки стратегии, но должны быть сопоставлены с стратегическим направлением, которое вытекает из стратегического процесса. Стратегия, которая последовательна, согласована и приспособлена к инновациям столь же важна, как и динамические способности для достижения конкурентного преимущества. Следовательно, пока стратегия и способности могут быть обособлены, в практическом плане их необходимо разрабатывать и внедрять вместе.

Концепция динамических способностей Д. Тиса является развитием так называемой ресурсной теории (Resource-based view) [14, 3, 8, 13], одной из новейших современных организационных технологий, появившихся в начале 90-х годов XX-го века, и, основанной на представлении предприятия, как совокупности ресурсных групп и выделении ресурсов, которые являются ключевыми компетенциями, за счет которых достигаются конкурентные преимущества, а также передаче неспецифических для конкретного предприятия функций бизнеса сторонним организациям.

Путем сопоставления содержательной компоненты организационной гибкости со структурой динамических способностей делается вывод, что в основе организационной гибкости лежат главным образом два взаимозависимых элемента динамически способного предприятия: предпринимательское управление, потенциально умеющее, сочетать и рекомбинировать технологии, а также гибкие структуры, которые

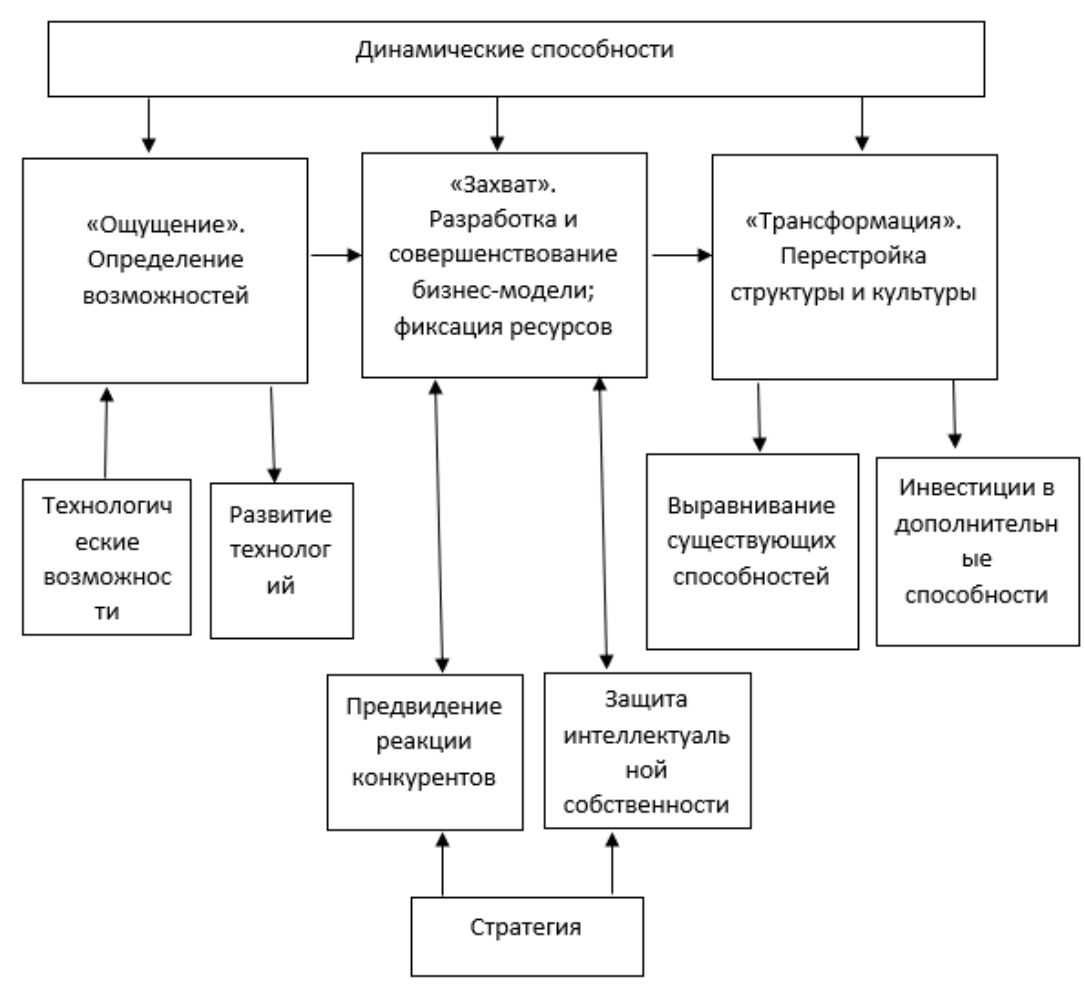

Рисунок 1. Упрощенная структура динамических способностей [12] 
могут быть быстро изменены.

Более детальное изучение способностей промышленного предприятия позволяет точнее идентифицировать место организационной гибкости среди прочих способностей, находящихся в арсенале предприятия. Д.Коллиз [1] предложил иерархию способностей предприятия, основанием которой служат операционные (функциональные) способности, являющиеся необходимыми для выживания компании и поддержания ключевых бизнес-процессов. Следующий уровень - уровень улучшений, связанный с совершенствованием организационных бизнеспроцессов, занимают динамические способности. Верхний уровень в иерархии является креативным, отражающим способности предприятия, относящиеся к умениям разрабатывать новые стратегии быстрее, чем конкуренты, управляя ценными ресурсными группами. Соответственно, ключевые компетенции находятся на этом уровне. Примечательно, что Б. Окстоби [7] с коллегами выделяют ключевую динамическую способность, понимая под ней способность к организационным изменениям, которая является «общей по отношению ко всем другим динамическим способностям, встроенным в организацию». В свою очередь, М. Джавидан [4] рассматривает следующую иерархию компетенций. В нижней части иерархии находятся ресурсы, которые являются строительными блоками компетенций. Способности занимают второй уровень иерархии и относятся к умению предприятия использовать свои ресурсы. Их отличительной чертой является функциональная основа. Далее на третьем уровне расположились компетенции, представляющие собой кроссфункциональную интеграцию и координацию способностей. Высший уровень иерархии соответствует ключевым компетенциям, представляющие собой навыки и области знаний, которые являются результатом интеграции и гармонизации компетенций. Основные особенности данной иерархии заключаются в том, что последующий уровень является результатом синергии элементов на более низком уровне, а также каждый уровень включает в себя более высокий показатель добавленной стоимости для компании.

Некоторые исследователи, рассматривающие концепцию динамических способностей Д.Тиса в качестве эталонной структуры, идентифицировали организационную гибкость как одну из ключевых динамических способностей для организаций в целях достижения устойчи- вых конкурентных преимуществ [9] и выживания в высокодинамичных средах [6]. Д.Ли [5] с соавторами концептуализируют организационную гибкость как двумерную динамическую способность с предпринимательским (или наступательным) и адаптивным (или оборонительным) аспектом. И.Доз и М. Косонен [2] определяют организационную гибкость как «более высокого порядка динамическую способность, которая строится с течением времени». Развивая различные интерпретации данного понятия, и, опираясь на экспликацию в разрезе динамических способностей, можно предположить, что эта способность является наиболее эффективной относительно достижения и приумножения конкурентоспособности.

Таким образом, на основании иерархии организационных способностей предприятия Д.Коллиза, и, руководствуясь общими принципами, заложенными в иерархию компетенций М.Джавидана, модель развития способностей предприятия в условиях непредвиденных, быстротечных изменений и высокой доли неопределенности деловой среды, по мнению автора исследования, имеет вид (рисунок 2).

Предприятия должны быть готовы к динамичному сотрудничеству, чтобы обеспечить быструю и эффективную обработку заказов. Выполнение конкретного требования достигается с помощью определенной комбинации ключевых компетенций предприятий-партнеров, формирующих уникальную способность предприятия - организационную гибкость на базе коллаборативной сетевой платформы (рисунок 3).

Подводя итоги, перечислим основные выводы:

1) организационная гибкость - это способность предприятия ощущать изменения деловой среды и эффективно реагировать на них;

2) рассматривая организационную гибкость в структуре динамических способностей, делается вывод о необходимости достижения гармонии организационной гибкости с требованиями деловой среды и стратегии фирмы. Ключевая особенность организационной гибкости заключается в успешном управлении неопределенностью;

3) организационная гибкость представляет собой интеграцию ключевых компетенций предприятий-партнеров производственной сети. Следовательно, организационная гибкость - это наивысшее развитие динамических способностей, как результат интеграции ключевых компетенций. 


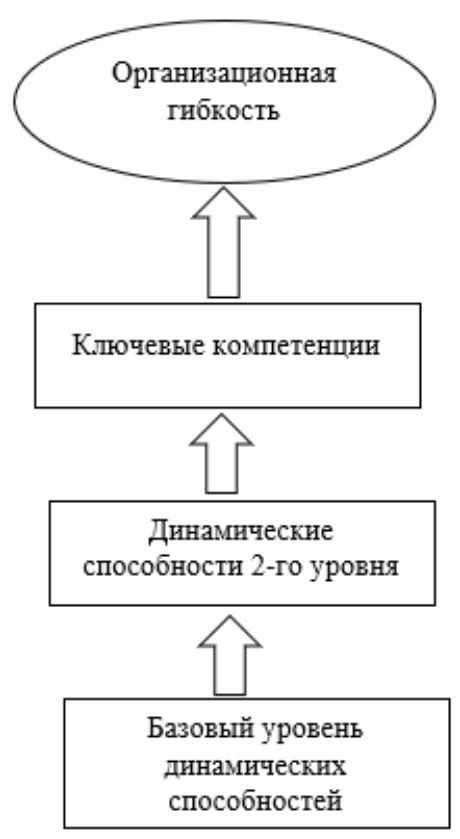

Рисунок 2. Модель развития способностей предприятия

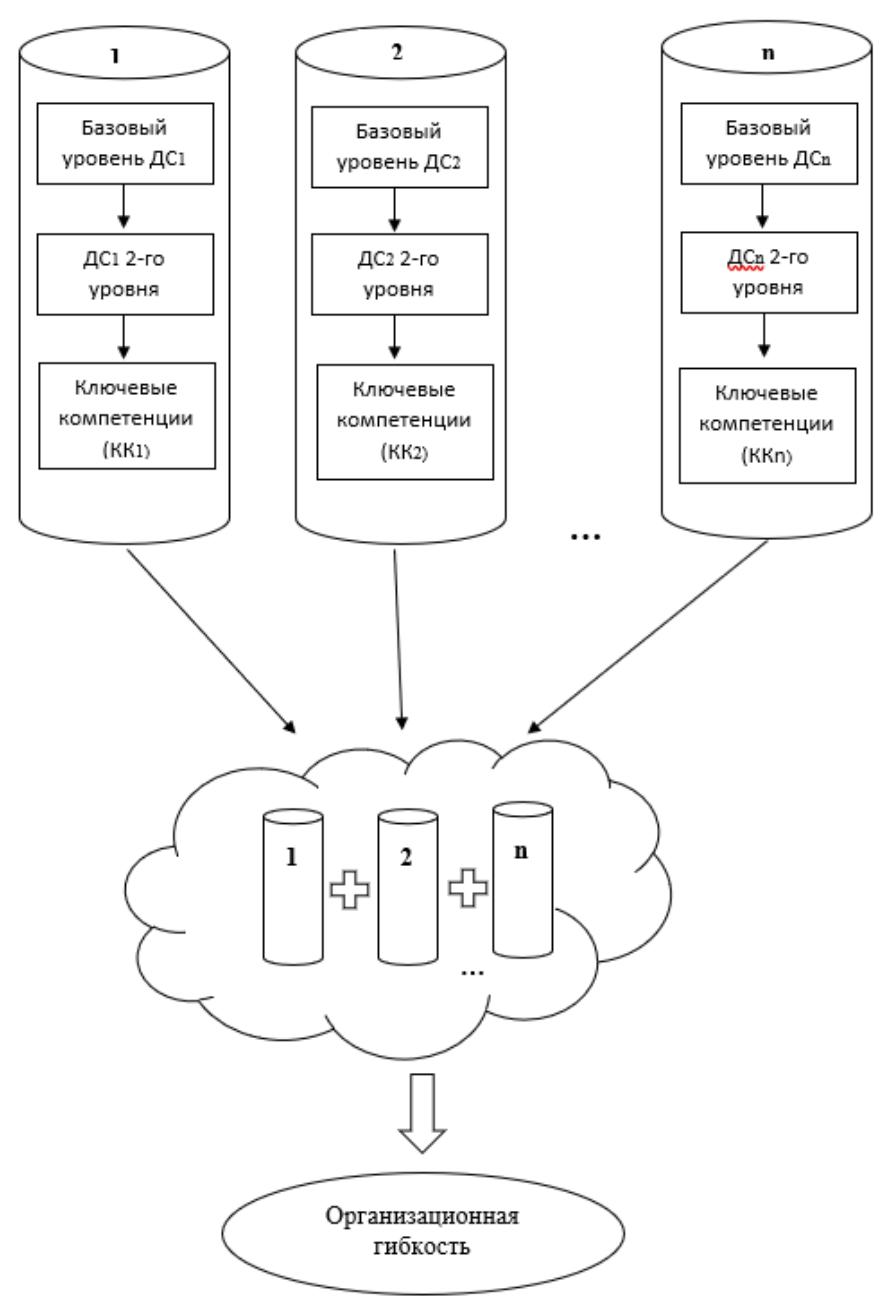

Рисунок 3. Формирование организационной гибкости 


\section{Библиографический список}

1. Collis, D. J. Research note: How Valuable are Organizational Capabilities? Strategic Management Journal. Vol. 15, N.8, 1994, pp. 143-152.

2. Doz Y. and Kosonen M. Fast Strategy: How Strategic Agility Will Help You Stay Ahead of the Game. Harlow, England: Pearson/Longman, 2008. p. 65.

3. Grant, R.M. The Resource-Based Theory of Competitive Advantage / R. M. Grant // Californian Management Review, 1991, N. 33 (3). P. 114-135.

4. Javidan M. Core Competence: What does it Mean in Practice? Long Range Planning. Vol. 31, № 1, 1998 , pp. 60-71.

5. Lee D, Sambamurthy V, Lim K, Wei KK. The moderating effects of environmental dynamism on the links between IT management and agility: A moderated mediation analysis, Working paper, University of Massachusetts, Boston, 2003.

6. Nijssen, M., and Paauwe, J. HRM in turbulent times: How to achieve organizational agility? International Journal of Human Resource Management. 23(16), 2012, pp. 3315-3335.

7. Oxtoby, B., McGuinness T., Morgan R.E. Developing Organisational Change Capability. European Management Journal, Vol.20, No.3, 2002, pp.310-320.

8. Prahalad, C.K., Hamel, G. (1990). Competence of the Corporation, Harvard Business Review, 68 (3), pp. 79-91.

9. Sambamurthy, V., Bharadwaj, A., and Grover, $V$. Shaping agility through digital options: Reconceptualizing the role of information technology in contemporary firms. MIS Quarterly. 27(2), 2003, pp. 237-263.

10. Teece, D., Peteraf, M. and Leih, S. Dynamic Capabilities and Organizational Agility: Risk, Uncertainty, and Strategy in the Innovation Economy. California Management Review. Vol. 58, No. 4, 2016, pp. 13-35.

11. Teece D.J. Explicating Dynamic Capabilities: The Nature and Microfoundations of (Sustainable) Enterprise Performance // Strategic Management Journal. № 28 (13). 2007, pp. 1319-1350.

12. Teece D. J. Business models and dynamic capabilities. Long Range Planning. № 51 (1), 2017, pp. 1-10.

13. Teece, D.J., Pisano, G., Shuen, A. (1997). Dynamic Capabilities and Strategic Management, Strategic Management Journal, 18 (7), pp. 509-533.

14. Wernerfelt, B.A. (1984). Resource-Based View of the Firm, Strategic Management Journal, 5 (2), pp. 171-180. 